\title{
Knowledge of self-care among type 2 diabetes patients in two states of Nigeria
}

\author{
Idongesit L. JACKSON, Maxwell O. ADIBE, Matthew J. OKONTA, Chinwe V. UKWE. \\ Received (first version):
}

\begin{abstract}
${ }^{*}$
Objective: To assess the knowledge of self-care practices, as well as factors responsible for such knowledge among type 2 diabetes patients in two states of Nigeria.

Methods: Descriptive, cross sectional survey research design was employed. The study was conducted on type 2 diabetes out-patients attending Endocrinology Clinic at the University of Uyo Teaching Hospital (UUTH) and University of Calabar Teaching Hospital (UCTH) between June 2012 and February 2013. The Diabetes Self-care Knowledge (DSCK-30) was used in evaluating knowledge of self-care practices. Socio-demographic information and respondents' opinion on the possible barrier(s) to knowledge of self-care were also obtained. Data were analysed using Microsoft Excel and SPSS version 14.0. Statistical significance for all analyses was defined as a $p$ value less than 0.05

Results: A total of 303 out of 380 questionnaires distributed were completed and returned (response rate $=79.7 \%)$. The majority of the study sample $(79.5 \%)$ had $70 \%$ or more overall knowledge level about self-care. Selfcare knowledge was associated with level of education $(p<0.001)$, monthly income $(p<0.001)$ and duration of diabetes $(p=0.008)$. Negative attitude to disease condition was the only factor associated with knowledge (chi-square value at one degree of freedom $=6.215 ; p=0.013$ ).

Conclusion: Diabetes self-care knowledge was generally high among the population studied. Educational status, monthly income, duration of diabetes and negative attitude to disease condition predicted knowledge level.
\end{abstract}

Keywords: Health Knowledge, Attitudes, Practice; Self Care; Diabetes Mellitus, Type 2; Nigeria

\footnotetext{
Idongesit L. JACKSON. M.Pharm. Lecturer, Department of Clinical Pharmacy and Biopharmacy, University of Uyo. Uyo (Nigeria). idyjack2@yahoo.com

Maxwell O. ADIBE. (PhD): Lecturer, Department of Clinical Pharmacy and Pharmacy

Management, University of Nigeria. Nsukka (nigeria) maxolpharmacia@yahoo.com

Matthew J. OKONTA. Professor and Head of Department of Clinical Pharmacy and Pharmacy Management, University of Nigeria. Nsukka (Nigeria).

okojema@yahoo.com

Chinwe V. UKWE. Professor, Department of Clinical

Pharmacy and Pharmacy Management,

University of Nigeria. Nsukka (Nigeria).

chinwe.ukwe@unn.edu.ng
}

\section{INTRODUCTION}

Diabetes is a chronic progressive endocrinological disorder of carbohydrate, protein and lipid metabolism, characterized by elevated levels of glucose in the blood (hyperglycaemia) due mainly to absolute insulin deficiency (in type 1) or relative deficiency and insulin resistance (in type 2)

Globally, the number of people with type 2 diabetes is rising rapidly. This rise is associated with population growth, economic development, ageing populations, increasing urbanisation, dietary changes, obesity, reduced physical activity and changes in other lifestyle patterns. ${ }^{1,2}$ The International Diabetes Federation ${ }^{3}$ indicated a global estimate of more than 371 million people living with diabetes in 2012, with a prevalence of $8.3 \%$. Half of these people are not even aware that they have it. In Africa, with diabetes census of 15 million, cases are expected to almost double over the next 20 years. Sadly, about $81.2 \%$ of people in this region do not even know they are living with the disease. ${ }^{3}$ In Nigeria with a national prevalence of $4.83 \%$, over 3 million people are currently living with diabetes. $^{3}$

Diabetes is one of the major causes of morbidity and mortality; it has a significant impact on the patients' quality of life, productivity and involves enormous health costs for virtually every society. ${ }^{4}$ One in twenty adult deaths in developing countries is diabetes-related ${ }^{5}$, with Africa having the highest mortality rate due to diabetes. ${ }^{3}$ Complications due to diabetes are implicated in disability, increased cost of care, reduced quality of life and death. ${ }^{6}$ Most of these medical problems can however, be prevented with proper self-care, as emphasised in the standard treatment guidelines of Nigeria. ${ }^{7}$

For effective management of diabetes, patients must be actively involved in their care: this requires performance of many complex self-care behaviours including lifestyle modifications (such as dietary control, regular exercise and psychosocial coping skills) and medical self-care (medication use and self-monitoring of blood glucose (SMBG)). Importantly, adequate self-care needs to persist over time if it is going to lessen complications and prolong life. ${ }^{8}$ The increase in severity of diabetes every year has been linked to patient's lack of knowledge and practice of proper self-care. ${ }^{9}$ According to Inzucchi ${ }^{10}$, a well-informed patient will have the best advantage to attain and maintain glycaemic and cardiovascular risk factor control. Consequently, poor knowledge of self-care can cause poor long-term metabolic control which may lead to the development of diabetic complications 
such as retinopathy, nephropathy, neuropathy, and atherosclerotic changes. Thus, patients require education about the various aspects of self-care, ranging from general lifestyle advice to knowledge about the medicine they are prescribed (cardiovascular risk factors like smoking, obesity; regular medical and ophthalmological examinations, foot care, diet, etc). ${ }^{11}$

Due to its extreme importance, an annual assessment of patients' skills and knowledge has been recommended by The American Diabetes Association. ${ }^{12}$ This can be easily made by administering a written or oral evaluation with each outpatient visit. A tool such as the 30 -item Diabetes Self-care Knowledge (DSCK-30) that can be selfadministered can facilitate this process both for patients who visit their health care practitioner regularly and for those who do not. Therefore, the purpose of this study was to assess the knowledge of self-care practices as well as factors affecting such knowledge among type 2 diabetes patients in two states of Nigeria.

\section{METHODS}

\section{Ethical Approval}

Ethical approval for this study was granted by the Health Research Ethics Committees of University of Uyo Teaching Hospital and University of Calabar Teaching Hospital.

\section{Data Collection}

The questionnaire, which was available in English language, was used for data collection. The instrument comprised three sections: the first section consisted of socio-demographic information; the second sought the respondent's opinion on the possible barrier(s) to knowledge of self-care; while the last section included questions on self-care the DSCK-30. The DSCK-30 is a thirty-item tool validated by Adibe and colleagues ${ }^{13}$ that measures type 2 diabetes knowledge of self-care. It is a structured measure with response choices of 'yes' and 'no.'

The questionnaires were administered to respondents from whom informed consent had been obtained, and collected on the spot after completion. This was done on the various clinic days between June, 2012 and February, 2013, ensuring that no respondent was surveyed more than once. Some of the respondents (about 30\%) could not read or understand English necessitating translation into the local language (Efik-lbibio) of the people. Only the principal investigator who could speak the local language of the people in this region was involved in the translation. Patients with poor sights were also assisted with reading and completing the survey.

\section{Inclusion/Exclusion Criteria}

Type 2 diabetic patients who are up to 18 years and above, who have been taking anti-diabetic medications for at least one month prior to the study, and are willing to participate were included in the study. While those with type 2 diabetes that were too ill to participate, pregnant, newly diagnosed (less than 1 month), or yet to be placed on medications were excluded.

\section{Data management}

Data were coded into Microsoft Excel, and analysed using Statistical Package for the Social Sciences (SPSS) Window version 14.0.

Scores on the DSCK-30 were computed for each participant. A point was awarded for each correct response, and zero for a wrong one. The total score was represented as a percentage. Knowledge of self-care was categorised as either low (if less than $70 \%$ ), or high (if equal to, or greater than $70 \%$ ). The mean performance of each item on the DSCK-30 was computed by summing up the responses from all participants and converting to percentages. For a knowledge question incorrectly answered by more than $30 \%$ of patients (i.e., \% correctness $<70 \%$ ), the corresponding knowledge area was identified as representing a deficiency.

Descriptive statistics (frequency) was used to summarize patients' socio-demographic data and evaluate distribution of responses. Chi-square was used to investigate association between predictors (factors) and knowledge level. Wilcoxon-MannWhitney and Kruskal-Wallis tests were performed appropriately to identify any association between patient characteristics and knowledge level. Statistical significance for all analyses was defined as a $p$ value less than 0.05 .

\section{RESULTS}

\section{Respondents' characteristics}

A total of 303 out of 380 questionnaires distributed were completed and returned, giving a response rate of $79.7 \%$. The number of males was 132 $(43.6 \%)$, while that of females was $171(56.4 \%)$. Most of the respondents $(38.9 \%)$ were in the age group of $50-59$ years. Almost half of them (49.5\%) had tertiary education, while $7(2.3 \%)$ said they had no formal education. More than three-quarter of them $(78.9 \%)$ were married. A hundred and four $(34.3 \%)$ were civil servants and $84(27.7 \%)$ were retired. Most of the patients (37.3\%) earned below NGN30,000 per month, and have had diabetes for $1-5$ years $(34.7 \%)$. One hundred and sixty one $(53.1 \%)$ respondents did not have any family members, relatives or friends with diabetes while $142(46.9 \%)$ said they did. Majority of them do not smoke (98.7\%) nor take alcohol (83.8\%). A summary of the demographic characteristics of respondents is given in Table 1.

\section{Knowledge level of respondents}

The majority of the study sample, 241 (79.5\%) had a high $(70 \%$ or over) overall knowledge level about self-care while the rest $(20.5 \%)$ showed low $(<70 \%)$ overall knowledge.

Table 2 shows that self-care knowledge was associated with level of education $(p=0.000)$, monthly income $(p=0.000)$ and duration of diabetes $(p=0.008)$. Patients with no formal education were 


\begin{tabular}{|c|c|c|}
\hline & $\mathrm{N}$ & $\%$ \\
\hline \multicolumn{3}{|l|}{ Gender } \\
\hline Male & 132 & 43.6 \\
\hline Female & 171 & 56.4 \\
\hline \multicolumn{3}{|l|}{ Age (Years) } \\
\hline$<30$ & 7 & 2.3 \\
\hline $30-39$ & 19 & 6.3 \\
\hline $40-49$ & 70 & 23.1 \\
\hline $50-59$ & 118 & 38.9 \\
\hline $60-69$ & 65 & 21.5 \\
\hline$>69$ & 24 & 7.9 \\
\hline \multicolumn{3}{|l|}{ Education } \\
\hline No formal education & 7 & 2.3 \\
\hline Primary & 65 & 21.5 \\
\hline Secondary & 81 & 26.7 \\
\hline Tertiary & 150 & 49.5 \\
\hline \multicolumn{3}{|l|}{ Marital Status } \\
\hline Single & 7 & 2.3 \\
\hline Married & 239 & 78.9 \\
\hline Widowed & 47 & 15.5 \\
\hline Divorced & 10 & 3.3 \\
\hline \multicolumn{3}{|l|}{ Occupation } \\
\hline Civil Servant & 104 & 34.3 \\
\hline Self employed/Business & 94 & 31.0 \\
\hline Retired & 84 & 27.7 \\
\hline Student & 4 & 1.3 \\
\hline Clergy & 4 & 1.3 \\
\hline Unemployed & 13 & 4.3 \\
\hline \multicolumn{3}{|l|}{ Monthly Income (NGN)* } \\
\hline$<30,000$ & 113 & 37.3 \\
\hline $30,000-70,000$ & 98 & 32.3 \\
\hline$>70,000$ & 74 & 24.4 \\
\hline Unsalaried & 18 & 5.9 \\
\hline \multicolumn{3}{|l|}{ Duration of diabetes (Years) } \\
\hline - & 33 & 10.9 \\
\hline $1-5$ & 105 & 34.7 \\
\hline $6-10$ & 84 & 27.7 \\
\hline$>10$ & 81 & 26.7 \\
\hline \multicolumn{3}{|l|}{$\begin{array}{l}\text { Do you have family members, } \\
\text { relatives or friends with diabetes? }\end{array}$} \\
\hline No & 161 & 53.1 \\
\hline Yes & 142 & 46.9 \\
\hline \multicolumn{3}{|l|}{ Current smoker } \\
\hline No & 299 & 98.7 \\
\hline Yes & 4 & 1.3 \\
\hline \multicolumn{3}{|l|}{ Alcohol intake } \\
\hline $\begin{array}{l}\text { No } \\
\text { Yes }\end{array}$ & $\begin{array}{c}254 \\
49\end{array}$ & $\begin{array}{l}83.8 \\
16 ?\end{array}$ \\
\hline co & & \\
\hline
\end{tabular}

less knowledgeable (57.1\%) than those with tertiary education $(84.7 \%)$. Those with a monthly income of less than NGN30,000 were likely to be less knowledgeable than those earning above NGN70,000 (72.6\% as compared with $87.8 \%)$. Those without any income had approximately the same knowledge level as those with monthly income of less than NGN30,000. Respondents who have had diabetes for over 10years were found to be more knowledgeable $(85.2 \%)$ than those who have had the disease for less than 1 year, between $1-5$ years, and between $6-10$ years $(63.6 \%, 78.1 \%$, and $82.1 \%$ respectively). Females were likely to be more knowledgeable than men; however this did not reach statistical significance $(p=0.655)$. This study also shows that patients who were single were more knowledgeable $(85.7 \%)$ than those who were married $(81.2 \%)$, widowed $(74.5 \%)$, and divorced $(60.0 \%)$; although statistical significance was not attained $(p=0.115)$. No significant association was found between knowledge and age, occupation, having family members, relatives or friends with diabetes, being a current smoker, or taking alcohol.

\section{Knowledge gaps / DSCK-30 Item Performance}

Table 2 shows the general performance of respondents on the knowledge items, as well as the relationship between socio-demographic data and knowledge levels across the three components of the scale. Although the general performance of respondents on the knowledge questions was high, the highest scores were obtained for items of component 2 (adherence to self-care practices) while the least scores were reported for items of component 1 (modifiable lifestyles). This trend is reflected in the median values (Table 2 ) as well as the $\% \mathrm{C}$ values (Table 3 ).

As shown in Table 3, six items of component 1, and one item each of components 2 and 3 revealed knowledge gaps (\% correctness $<70 \%$ ).

\section{Factors affecting self-care knowledge}

Out of the five factors listed that could affect knowledge of diabetes self-care, only one factor negative attitude to disease condition - met statistical significance (chi-square value at one degree of freedom $=6.215 ; p=0.013$ ). For this factor, there is a significant difference between the patients' response and knowledge levels. However, no significant difference exists between patients' self-care knowledge level and their opinion regarding other likely causes of poor knowledge (Table 4).

\section{DISCUSSION}

\section{Patients' characteristics}

In this study, the highest number of type 2 diabetics were in the age range of $50-59$ years $(n=118)$ followed by those in the age range of $40-49$ years $(n=70)$. This is in line with IDF $^{6}$ which reported that the greatest number of people with diabetes are between 40-59 years. According to King and colleagues $^{2}$, worldwide overall diabetes prevalence is higher in men, but there are more women with diabetes than men, probably due to the combined effect of a greater number of elderly women than men in most populations and the increasing prevalence of diabetes with age. A recent survey found the male and female prevalence of the disease to be $9.6 \%$ and $11.2 \%$ respectively. ${ }^{14}$ It is therefore not surprising that women constituted $56.4 \%$ of the present study sample while men $43.6 \%$. Previous studies. ${ }^{13,15,16}$ also reported similar gender distribution. $73.3 \%$ of the respondents had had diabetes for less than 10 years, while only $26.7 \%$ had had it for more than 10 years. A possible reason for this is that either diabetes is associated with recent changes in dietary habits and lifestyle modifications, or it is a reflection of a high patient mortality. ${ }^{17}$

\section{Knowledge gaps}

Although the general performance of the knowledge items was high, areas such as glycosylated haemoglobin (HbA1c), physical activities, hypoglycaemic symptoms, and medication-related items performed relatively poorly. 


\begin{tabular}{|c|c|c|c|c|c|c|c|c|c|c|c|c|}
\hline \multirow{2}{*}{ Characteristics } & \multicolumn{3}{|c|}{ Overall (DSCK-30) } & \multicolumn{3}{|c|}{$\begin{array}{l}\text { Modifiable Lifestyles } \\
\text { (Component 1) }\end{array}$} & \multicolumn{3}{|c|}{$\begin{array}{l}\text { Adherence to Self-care } \\
\text { (Component 2) }\end{array}$} & \multicolumn{3}{|c|}{$\begin{array}{c}\text { Consequences of Uncontrolled } \\
\text { Blood Sugar Level (Component 3) }\end{array}$} \\
\hline & $\begin{array}{l}\text { High Kn. } \\
\mathrm{n}(\%)\end{array}$ & $\begin{array}{c}\text { Median } \\
(\text { Min-Max })\end{array}$ & P-value & $\begin{array}{l}\text { High Kn. } \\
\mathrm{n}(\%)\end{array}$ & $\begin{array}{l}\text { Median } \\
(\text { Min-Max) }\end{array}$ & P-value & $\begin{array}{l}\text { High Kn. } \\
\mathrm{n}(\%)\end{array}$ & $\begin{array}{l}\text { Median } \\
(\text { Min-Max }) \\
\end{array}$ & P-value & $\begin{array}{l}\text { High Kn. } \\
\mathrm{n}(\%)\end{array}$ & $\begin{array}{l}\text { Median } \\
\text { (Min-Max) } \\
\end{array}$ & P-value \\
\hline $\begin{array}{r}\text { Male } \\
\text { Female }\end{array}$ & $\begin{array}{l}103(78.0) \\
138(80.7) \\
\end{array}$ & $\begin{array}{c}76.7 \\
(33.3-100.0) \\
76.7 \\
(46.7-93.3) \\
\end{array}$ & 0.655 & $\begin{array}{l}72(54.5) \\
91(53.2)\end{array}$ & $\begin{array}{c}72.2 \\
(38.9-94.4) \\
72.2 \\
(33.3-88.9) \\
\end{array}$ & 0.628 & $\begin{array}{l}103(78.0) \\
137(80.1) \\
\end{array}$ & $\begin{array}{c}87.5 \\
(25.0-100.0) \\
87.5 \\
(37.5-100.0) \\
\end{array}$ & 0.776 & $\begin{array}{l}113(85.6) \\
147(86.0) \\
\end{array}$ & $\begin{array}{c}75.0 \\
(26.0-100.0) \\
75.0 \\
(25.0-100.0) \\
\end{array}$ & 0.829 \\
\hline $\begin{array}{rr}\text { Age (Years) } & <30 \\
& 30-39 \\
& 40-49 \\
50-59 \\
60-69 \\
>69\end{array}$ & $\begin{array}{c}6(85.7) \\
15(78.9) \\
51(72.9) \\
101(85.6) \\
48(73.8) \\
20(83.3)\end{array}$ & $\begin{array}{c}76.7 \\
(56.7-90.0) \\
76.7 \\
(43.3-90.0) \\
76.7 \\
(33.3-93.3) \\
80.0 \\
(53.3-93.3) \\
76.7 \\
(43.3-100.0) \\
76.7 \\
(53.3-90.0) \\
\end{array}$ & 0.333 & $\begin{array}{l}3(42.9) \\
12(63.2) \\
38(54.3) \\
70(59.3) \\
28(43.1) \\
12(50.0) \\
\end{array}$ & $\begin{array}{c}66.7 \\
(50.0-77.8) \\
72.2 \\
(44.4-83.3) \\
72.2 \\
(33.3-83.3) \\
72.2 \\
(50.0-88.9) \\
66.7 \\
(44.4-94.4) \\
69.4 \\
(44.4-83.3) \\
\end{array}$ & 0.412 & $\begin{array}{l}4(57.1) \\
14(73.7) \\
50(71.4) \\
99(83.9) \\
53(81.5) \\
20(83.3) \\
\end{array}$ & $\begin{array}{c}87.5 \\
(37.5-100.0) \\
75.0 \\
(25.0-100.0) \\
75.0 \\
(25.0-100.0) \\
87.5 \\
(37.5-100.0) \\
87.5 \\
(37.5-100.0) \\
87.5 \\
(50.0-100.0) \\
\end{array}$ & 0.227 & $\begin{array}{l}7(100.0) \\
17(89.5) \\
60(85.7) \\
106(89.8) \\
51(78.5) \\
19(79.2) \\
\end{array}$ & $\begin{array}{c}75.0 \\
(75.0-100.0) \\
75.0 \\
(50.0-100.0) \\
75.0 \\
(25.0-100.0) \\
75.0 \\
(25.0-100.0) \\
75.0 \\
(25.0-100.0) \\
75.0 \\
(25.0-100.0) \\
\end{array}$ & 0.640 \\
\hline $\begin{array}{r}\text { Education } \\
\text { None } \\
\text { Primary } \\
\text { Secondary } \\
\text { Tertiary }\end{array}$ & $\begin{array}{c}4(57.1) \\
47(72.3) \\
63(77.8) \\
127(84.7)\end{array}$ & $\begin{array}{c}70.0 \\
(56.7-100.0) \\
73.3 \\
(46.7-93.3) \\
76.7 \\
(43.3-90.0) \\
80.0 \\
(33.3-96.7) \\
\end{array}$ & 0.000 & $\begin{array}{c}2(28.6) \\
30(46.2) \\
33(40.7) \\
98(65.3)\end{array}$ & $\begin{array}{c}66.7 \\
(50.0-94.4) \\
66.7 \\
(44.4-83.3) \\
66.7 \\
(44.4-83.3) \\
72.2 \\
(33.3-88.9) \\
\end{array}$ & 0.001 & $\begin{array}{c}5(71.4) \\
45(69.2) \\
66(81.5) \\
124(82.7)\end{array}$ & $\begin{array}{c}87.5 \\
(62.5-100.0) \\
75.0 \\
(37.5-100.0) \\
87.5 \\
(25.0-100.0) \\
87.5 \\
(25.0-100.0) \\
\end{array}$ & 0.025 & $\begin{array}{c}4(57.1) \\
52(80.0) \\
67(82.7) \\
137(91.3)\end{array}$ & $\begin{array}{c}75.0 \\
(25.0-100.0) \\
75.0 \\
(25.0-100.0) \\
75.0 \\
(25.0-100.0) \\
75.0 \\
(25.0-100.0) \\
\end{array}$ & 0.116 \\
\hline $\begin{array}{r}\text { Marital Status } \\
\text { Single } \\
\text { Married } \\
\text { Widowed } \\
\text { Divorced }\end{array}$ & $\begin{array}{c}6(85.7) \\
194(81.2) \\
35(74.5) \\
6(60.0)\end{array}$ & $\begin{array}{c}76.7 \\
(63.3-90.0) \\
80.0 \\
(33.3-100.0) \\
76.7 \\
(53.3-93.3) \\
71.7 \\
(60.0-90.0) \\
\end{array}$ & 0.115 & $\begin{array}{c}3(42.9) \\
136(56.9) \\
19(40.4) \\
5(50.0)\end{array}$ & $\begin{array}{c}66.7 \\
(55.6-83.3) \\
72.2 \\
(33.3-94.4) \\
66.7 \\
(44.4-88.9) \\
66.7 \\
(50.0-83.3)\end{array}$ & 0.382 & $\begin{array}{c}5(71.4) \\
190(79.5) \\
38(80.9) \\
7(70.0)\end{array}$ & $\begin{array}{c}87.5 \\
(50.0-100.0) \\
87.5 \\
(25.0-100.0) \\
75.0 \\
(37.5-100.0) \\
75.0 \\
(50.0-100.0) \\
\end{array}$ & 0.478 & $\begin{array}{c}7(100.0) \\
209(87.4) \\
38(80.9) \\
6(60.0)\end{array}$ & $\begin{array}{c}75.0 \\
(75.0-100.0) \\
75.0 \\
(25.0-100.0) \\
75.0 \\
(25.0-100.0) \\
75.0 \\
(25.0-100.0)\end{array}$ & 0.215 \\
\hline $\begin{array}{r}\text { Current Smoker } \\
\text { No } \\
\text { Yes } \\
\end{array}$ & $\begin{array}{c}237(79.3) \\
4(100.0) \\
\end{array}$ & $\begin{array}{c}76.7 \\
(33.3-100.0) \\
76.7 \\
(73.3-90.0) \\
\end{array}$ & 0.315 & $\begin{array}{c}161(53.8) \\
2(50.0) \\
\end{array}$ & $\begin{array}{c}72.2 \\
(33.3-94.4) \\
72.2 \\
(66.7-77.8) \\
\end{array}$ & 0.658 & $\begin{array}{c}236(78.9) \\
4(100.0) \\
\end{array}$ & $\begin{array}{c}87.5 \\
(25.0-100.0) \\
87.5 \\
(87.5-100.0) \\
\end{array}$ & 0.305 & $\begin{array}{c}256(85.6) \\
4(100.0) \\
\end{array}$ & $\begin{array}{c}75.0 \\
(25.0-100.0) \\
87.5 \\
(75.0-100.0) \\
\end{array}$ & 0.224 \\
\hline
\end{tabular}




\begin{tabular}{|c|c|c|c|c|c|c|c|c|c|c|c|c|}
\hline \multirow{2}{*}{ Characteristics } & \multicolumn{3}{|c|}{ Overall (DSCK-30) } & \multicolumn{3}{|c|}{$\begin{array}{l}\text { Modifiable Lifestyles } \\
\text { (Component 1) }\end{array}$} & \multicolumn{3}{|c|}{$\begin{array}{l}\text { Adherence to Self-care } \\
\text { (Component 2) }\end{array}$} & \multicolumn{3}{|c|}{$\begin{array}{c}\text { Consequences of Uncontrolled } \\
\text { Blood Sugar Level (Component 3) }\end{array}$} \\
\hline & $\begin{array}{l}\text { High Kn. } \\
\text { n (\%) }\end{array}$ & $\begin{array}{c}\text { Median } \\
(\text { Min-Max) }\end{array}$ & $P$-value & $\begin{array}{l}\text { High Kn. } \\
\mathrm{n}(\%)\end{array}$ & $\begin{array}{c}\text { Median } \\
\text { (Min-Max) }\end{array}$ & P-value & $\begin{array}{c}\text { High Kn. } \\
\mathrm{n}(\%)\end{array}$ & $\begin{array}{l}\text { Median } \\
\text { (Min-Max) }\end{array}$ & P-value & $\begin{array}{l}\text { High Kn. } \\
\mathrm{n}(\%)\end{array}$ & $\begin{array}{c}\text { Median } \\
(\text { Min-Max) } \\
\end{array}$ & P-value \\
\hline $\begin{array}{r}\text { Occupation } \\
\text { Civil Servant } \\
\text { Self-employed } \\
\text { Retired } \\
\text { Student } \\
\text { Unemployed } \\
\text { Clergy }\end{array}$ & $\begin{array}{l}82(78.8) \\
74(78.7) \\
69(82.1) \\
3(75.0) \\
9(69.2) \\
4(100.0)\end{array}$ & $\begin{array}{c}80.0 \\
(33.3-93.3) \\
76.7 \\
(43.3-100.0) \\
78.3 \\
(56.7-96.7) \\
75.0 \\
(63.3-83.3) \\
73.3 \\
(53.3-90.0) \\
88.3 \\
(80.0-93.3) \\
\end{array}$ & 0.085 & $\begin{array}{c}59(56.7) \\
48(51.1) \\
45(53.6) \\
1(25.0) \\
6(46.2) \\
4(100.0)\end{array}$ & $\begin{array}{c}72.2 \\
(33.3-88.9) \\
72.2 \\
(44.4-94.4) \\
72.2 \\
(50.0-88.9) \\
66.7 \\
(55.6-77.8) \\
66.7 \\
(44.4-77.8) \\
80.6 \\
(72.2-83.3) \\
\end{array}$ & 0.199 & $\begin{array}{c}83(79.8) \\
71(75.5) \\
70(83.3) \\
2(50.0) \\
10(76.9) \\
4(100.0)\end{array}$ & $\begin{array}{c}87.5 \\
(25.0-100.0) \\
87.5 \\
(25.0-100.0) \\
87.5 \\
(37.5-100.0) \\
68.8 \\
(62.5-100.0) \\
75.0 \\
(50.0-100.0) \\
93.8 \\
(75.0-100.0) \\
\end{array}$ & 0.551 & $\begin{array}{c}92(88.5) \\
79(84.0) \\
72(85.7) \\
4(100.0) \\
9(69.2) \\
4(100.0)\end{array}$ & $\begin{array}{c}75.0 \\
(25.0-100.0) \\
75.0 \\
(25.0-100.0) \\
75.0 \\
(25.0-100.0) \\
75.0 \\
(75.0-100.0) \\
75.0 \\
(25.0-100.0) \\
100.0 \\
(75.0-100.0) \\
\end{array}$ & 0.145 \\
\hline $\begin{array}{r}\text { Monthly Income }(\#) \\
<30,000 \\
30,000-70,000 \\
>70,000 \\
\text { Unsalaried }\end{array}$ & $\begin{array}{l}82(72.6) \\
81(82.7) \\
65(87.8) \\
13(72.2)\end{array}$ & $\begin{array}{c}76.7 \\
(33.3-96.7) \\
76.7 \\
(43.3-100.0) \\
83.3 \\
(56.7-93.3) \\
73.3 \\
(53.3-90.0) \\
\end{array}$ & 0.000 & $\begin{array}{c}53(46.9) \\
49(50.0) \\
53(71.6) \\
8(44.4)\end{array}$ & $\begin{array}{c}66.7 \\
(38.9-88.9) \\
69.4 \\
(33.3-94.4) \\
77.8 \\
(50.0-88.9) \\
66.7 \\
(44.4-77.8) \\
\end{array}$ & 0.001 & $\begin{array}{l}83(73.5) \\
81(82.7) \\
63(85.1) \\
13(72.2)\end{array}$ & $\begin{array}{c}87.5 \\
(25.0-100.0) \\
87.5 \\
(37.5-100.0) \\
87.5 \\
(37.5-100.0) \\
75.0 \\
(50.0-100.0) \\
\end{array}$ & 0.016 & $\begin{array}{l}93(82.3) \\
86(87.8) \\
68(91.9) \\
13(72.2)\end{array}$ & $\begin{array}{c}75.0 \\
(25.0-100.0) \\
75.0 \\
(25.0-100.0) \\
75.0 \\
(25.0-100.0) \\
75.0 \\
(25.0-100.0) \\
\end{array}$ & 0.223 \\
\hline $\begin{array}{l}\begin{array}{l}\text { Duration of Diabetes } \\
\text { (Years) }\end{array} \\
<1 \\
1-5 \\
6-10 \\
>10\end{array}$ & $\begin{array}{l}82(78.1) \\
69(82.1) \\
69(85.2)\end{array}$ & $\begin{array}{c}73.3 \\
(33.3-90.0) \\
76.7 \\
(43.3-96.7) \\
80.0 \\
(43.3-93.3) \\
80.0 \\
(53.3-100.0) \\
\end{array}$ & 0.008 & $\begin{array}{l}15(45.5) \\
50(47.6) \\
49(58.3) \\
49(60.5)\end{array}$ & $\begin{array}{c}66.7 \\
(38.9-83.3) \\
66.7 \\
(44.4-88.9) \\
72.2 \\
(33.3-88.9) \\
72.2 \\
(44.4-94.4) \\
\end{array}$ & 0.014 & $\begin{array}{l}25(75.8) \\
80(76.2) \\
65(77.4) \\
70(86.4)\end{array}$ & $\begin{array}{c}75.0 \\
(25.0-100.0) \\
75.0 \\
(25.0-100.0) \\
87.5 \\
(37.5-100.0) \\
87.5 \\
(37.5-100.0) \\
\end{array}$ & 0.130 & $\begin{array}{l}88(83.8) \\
73(86.9) \\
72(88.9)\end{array}$ & $\begin{array}{c}75.0 \\
(25.0-100.0) \\
75.0 \\
(25.0-100.0) \\
75.0 \\
(25.0-100.0) \\
75.0 \\
(25.0-100.0) \\
\end{array}$ & 0.497 \\
\hline $\begin{array}{l}\text { Relatives or Friends } \\
\text { with diabetes } \\
\text { No } \\
\text { Yes }\end{array}$ & $\begin{array}{l}127(78.9) \\
114(80.3)\end{array}$ & $\begin{array}{c}76.7 \\
(43.3-93.3) \\
76.7 \\
(33.3-100.0) \\
\end{array}$ & 0.217 & $\begin{array}{l}84(52.2) \\
79(55.6)\end{array}$ & $\begin{array}{c}72.2 \\
(44.4-88.9) \\
72.2 \\
(33.3-94.4) \\
\end{array}$ & 0.607 & $\begin{array}{l}126(78.3) \\
114(80.3)\end{array}$ & $\begin{array}{c}87.5 \\
(25.0-100.0) \\
87.5 \\
(25.0-100.0) \\
\end{array}$ & 0.113 & $\begin{array}{l}139(86.3) \\
121(85.2)\end{array}$ & $\begin{array}{c}75.0 \\
(25.0-100.0) \\
75.0 \\
(25.0-100.0) \\
\end{array}$ & 0.580 \\
\hline $\begin{array}{rr}\text { Alcohol Intake } & \\
& \text { No } \\
& \text { Yes }\end{array}$ & $\begin{array}{c}204(80.3) \\
37(75.5)\end{array}$ & $\begin{array}{c}76.7 \\
(46.7-100.0) \\
76.7 \\
(33.3-93.3) \\
\end{array}$ & 0.558 & $\begin{array}{l}137(53.9) \\
26(53.1)\end{array}$ & $\begin{array}{c}72.2 \\
(33.3-94.4) \\
72.2 \\
(38.9-88.9)\end{array}$ & 0.945 & $\begin{array}{c}206(81.1) \\
34(69.4)\end{array}$ & $\begin{array}{c}87.5 \\
(37.5-100.0) \\
87.5 \\
(25.0-100.0) \\
\end{array}$ & 0.265 & $\begin{array}{c}219(86.2) \\
41(83.7)\end{array}$ & $\begin{array}{c}75.0 \\
(25.0-100.0) \\
75.0 \\
(25.0-100.0) \\
\end{array}$ & 0.998 \\
\hline
\end{tabular}




\begin{tabular}{|c|c|c|c|}
\hline $\begin{array}{l}\text { Item } \\
\text { No. }\end{array}$ & Item (Question) & $\begin{array}{l}\text { Response } \\
\text { options }\end{array}$ & $\%$ correctness \\
\hline \multicolumn{3}{|c|}{ Component 1: Modifiable Lifestyles } & $\begin{array}{c}{ }^{\mathrm{a}} 73.76 \\
(35.97-97.69)\end{array}$ \\
\hline 1. & $\begin{array}{l}{ }^{\mathrm{b}} \text { Fasting blood sugar (FBS) test can be used to monitor } 2 \text { to } 3 \text { months blood sugar } \\
\text { control. }\end{array}$ & Yes; No & 35.97 \\
\hline 3. & $\begin{array}{l}\text { bonly the doctors should make plans on how a person with diabetes can achieve } \\
\text { his/her target goals. }\end{array}$ & Yes; No & 45.21 \\
\hline 4. & $\begin{array}{l}\text { Blood glucose level should be measured before and after every planned physical } \\
\text { activity. }\end{array}$ & Yes; No & 40.26 \\
\hline 5. & $\begin{array}{l}\text { Having physical activity for } 20-30 \text { minutes per session at least } 3 \text { days per week is } \\
\text { essential. (Example of physical activities: Brisk walking, house activities, climbing } \\
\text { staircase). }\end{array}$ & Yes; No & 90.10 \\
\hline 6. & ${ }^{\mathrm{b}}$ Regular exercise does not reduce the need for insulin or other diabetic drugs. & Yes; No & 43.56 \\
\hline 7. & Maintaining a healthy weight is not important in management of diabetes. & Yes; No & 77.89 \\
\hline 8. & $\begin{array}{l}\text { A person with diabetes should only ask for help when he/she feels sick from his/her } \\
\text { healthcare team. }\end{array}$ & Yes; No & 70.96 \\
\hline 17. & $\begin{array}{l}\text { At the initiation of insulin therapy for a person with diabetes who may require it, } \\
\text { appropriate advice on Self Blood Glucose Monitoring (SBGM) and diets should be } \\
\text { given to the person. }\end{array}$ & Yes; No & 92.74 \\
\hline 18. & $\begin{array}{l}\text { b There should be mutual agreement between a person with diabetes and the doctor if } \\
\text { he/she cannot change a particular lifestyle and afford his/her drugs. }\end{array}$ & Yes; No & 59.74 \\
\hline 19. & $\begin{array}{l}\text { A person with diabetes should take extra care of his/her feet especially when cutting } \\
\text { his/her toenails }\end{array}$ & Yes; No & 97.69 \\
\hline 20. & Tight elastic hose or socks are not bad for a person with diabetes. & Yes; No & 78.88 \\
\hline 21. & $\begin{array}{l}\text { A person with diabetes should take care of his/her teeth and brush and floss his/her } \\
\text { teeth every day. }\end{array}$ & Yes; No & 96.70 \\
\hline 23. & $\begin{array}{l}\text { No person should check blood sugar and blood pressure of a diabetic patient except } \\
\text { qualified medical doctor and other health personnel in the hospital. }\end{array}$ & Yes; No & 49.17 \\
\hline 24. & A person with diabetes should report any change in his eyesight to his doctor. & Yes; No & 97.69 \\
\hline 25. & $\begin{array}{l}\text { Self blood glucose monitoring (SBGM) allows doctor and other healthcare team to } \\
\text { gather data for treatment planning. }\end{array}$ & Yes; No & 91.75 \\
\hline 29. & $\begin{array}{l}\text { Monitoring blood pressure is not as important as monitoring blood glucose in a person } \\
\text { with diabetes. }\end{array}$ & Yes; No & 70.30 \\
\hline \multicolumn{3}{|c|}{ Component 2: Adherence to Self-care Practices } & $\begin{array}{c}{ }^{\mathrm{a}} 81.64 \\
(56.11-92.08)\end{array}$ \\
\hline 2. & $\begin{array}{l}\text { Dietary instructions should be written out, even if the person with diabetes is illiterate: } \\
\text { someone at home should be available to interpret it for him/her. }\end{array}$ & ${ }^{*}$ Yes; No & 83.83 \\
\hline 10. & $\begin{array}{l}\text { A person with diabetes taking diabetic medicines even when he/she feels good is } \\
\text { waste of money. }\end{array}$ & Yes; No & 91.75 \\
\hline 11. & Being drunk while on diabetic drugs is not a serious problem & Yes; No & 91.75 \\
\hline 12. & Diet and exercise are not as important as medication in control of diabetes. & Yes; No & 83.83 \\
\hline 13. & Instructions about drugs and other self-care practices must not be strictly followed. & Yes; No & 86.14 \\
\hline 14. & $\begin{array}{l}\text { Regular medical checkups are not essential when a person with diabetes is feeling } \\
\text { well. }\end{array}$ & Yes; No & 92.08 \\
\hline 15. & $\begin{array}{l}\left.{ }^{\mathrm{b}} \text { Taking low dose Aspirin (Vasoprin®, Emprin } ®\right) \text { tablet every day decreases risk of } \\
\text { having heart attack and stroke. }\end{array}$ & Yes; No & 56.11 \\
\hline 16. & ${ }^{\mathrm{b}}$ Diabetes Drugs are not taken throughout the life time of a person with diabetes. & Yes; No & 67.66 \\
\hline \multicolumn{3}{|c|}{ Component 3: Consequences of uncontrolled blood sugar level } & $\begin{array}{l}{ }^{\mathrm{a}} 77.15 \\
(33.99-95.05)\end{array}$ \\
\hline 22. & $\begin{array}{l}\text { If blood sugar is close to normal, a person with diabetes is likely to have more energy, } \\
\text { feel less thirsty and urinate less often. }\end{array}$ & Yes; No & 91.42 \\
\hline 27. & $\begin{array}{l}\text { bShaking, confusion, behavioural changes and sweating are signs of high blood } \\
\text { sugar. }\end{array}$ & Yes; No & 33.99 \\
\hline 28. & Prolonged high blood sugar level can cause eye problem or even blindness. & Yes; No & 95.05 \\
\hline 30. & $\begin{array}{l}\text { Prolonged uncontrolled blood sugar level can cause heart attack, stroke and kidney } \\
\text { problems. }\end{array}$ & Yes; No & 88.12 \\
\hline \multicolumn{3}{|c|}{ Overall Average Performance (range) } & $\begin{array}{c}77.52 \\
(33.99-97.69)\end{array}$ \\
\hline \multicolumn{4}{|c|}{ 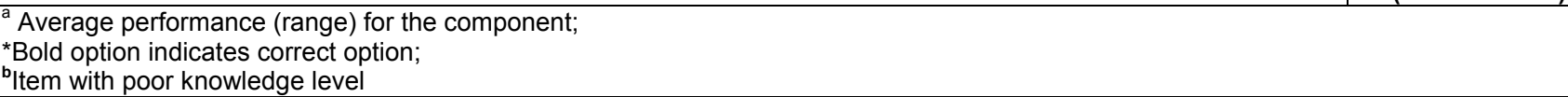 } \\
\hline
\end{tabular}

The items that were unfamiliar and newer (physical activities, SMBG, and $\mathrm{HbA} 1 \mathrm{c}$ ) constituted component 1 , whereas those associated with more traditional aspects (diet and medication-related items) of self-care management constituted component $2 .^{13}$ Thus, patients scored generally higher in items that were more familiar but did not score as high in the relatively new and unfamiliar knowledge items of the first component. 


\begin{tabular}{|c|c|c|c|c|c|}
\hline \multirow{2}{*}{ Factors Affecting Knowledge } & \multirow{2}{*}{ Response } & \multirow{2}{*}{$X *(\%)$} & \multicolumn{2}{|c|}{ Knowledge Level: n (\%) } & \multirow{2}{*}{$p$-value } \\
\hline & & & Low & High & \\
\hline $\begin{array}{l}\text { Lack of time to read about diabetes, or } \\
\text { attend diabetes education programmes }\end{array}$ & $\begin{array}{l}\text { Yes } \\
\text { No }\end{array}$ & $\begin{array}{l}173(57.1) \\
130(42.9)\end{array}$ & $\begin{array}{l}39(22.5) \\
23(17.7)\end{array}$ & $\begin{array}{l}134(77.5) \\
107(82.3)\end{array}$ & 0.300 \\
\hline $\begin{array}{l}\text { Lack of access to diabetic education and } \\
\text { regular updates }\end{array}$ & $\begin{array}{l}\text { Yes } \\
\text { No }\end{array}$ & $\begin{array}{l}193(63.7) \\
110(36.3)\end{array}$ & $\begin{array}{l}43(22.3) \\
19(17.3) \\
\end{array}$ & $\begin{array}{l}150(77.7) \\
91(82.7) \\
\end{array}$ & 0.299 \\
\hline Inadequate training on diabetes self-care & $\begin{array}{l}\text { Yes } \\
\text { No }\end{array}$ & $\begin{array}{l}176(58.1) \\
127(41.9)\end{array}$ & $\begin{array}{l}35(19.9) \\
27(21.3)\end{array}$ & $\begin{array}{l}141(80.1) \\
100(78.7) \\
\end{array}$ & 0.770 \\
\hline $\begin{array}{l}\text { The amount of time a patient receives from } \\
\text { a doctor in clinic }\end{array}$ & $\begin{array}{l}\text { Yes } \\
\text { No }\end{array}$ & $\begin{array}{l}150(49.5) \\
153(50.5)\end{array}$ & $\begin{array}{l}25(16.7) \\
37(24.2)\end{array}$ & $\begin{array}{l}125(83.3) \\
116(75.8)\end{array}$ & 0.105 \\
\hline Negative attitude to disease condition & $\begin{array}{l}\text { Yes } \\
\text { No }\end{array}$ & $\begin{array}{l}160(52.8) \\
143(47.2)\end{array}$ & $\begin{array}{l}24(15.0) \\
38(26.6)\end{array}$ & $\begin{array}{l}136(85.0) \\
105(73.4)\end{array}$ & ${ }^{d} 0.013$ \\
\hline
\end{tabular}

Studies have shown that medication knowledge is positively correlated with medication adherence. ${ }^{18-20}$ Thus, patients who do not know the benefits of low dose aspirin may easily skip doses, particularly as it is a very cheap drug. Lower levels of knowledge regarding the health benefits of aspirin were reported in earlier studies. ${ }^{15,21}$ Although most patients knew that diabetes drugs are taken for life, some did not. An even lower knowledge level was reported in a study by Adisa and colleagues. ${ }^{15} \mathrm{~A}$ possible reason for this knowledge deficiency is that some diabetes patients believe that diabetes can be cured after some time on anti-diabetic drugs, or with the use of herbs, particularly the bitter ones. ${ }^{22} \mathrm{~A}$ high rate of complementary / alternative medicine use has been reported among people with diabetes. ${ }^{23-26}$ Health care providers should constantly educate patients about the goals of therapy, the purpose of each drug prescribed, the importance of adhering to every prescribed medication as well as the consequences of nonadherence. Patients should be taught and constantly reminded that diabetes cannot be cured but can be well-managed if adherence to treatment is high.

In this study, most patients admitted that they experience excessive sweating, shaking, behavioural changes, and confusion. They however, erroneously attributed these to hyperglycaemia. Previous studies have reported inability of patients to identify hypoglycaemic symptoms ${ }^{13,21,27,28}$, indicating that diabetes educators/care providers should place more emphasis on the symptoms of hypoglycaemia and those of hyperglycaemia with the appropriate managements.

In line with earlier studies ${ }^{13,21}$, this research reveals that majority of the respondents erroneously thought that FBS test can be used to monitor 2 to 3 months blood sugar control. This may be due, in part, to the fact that most of the respondents in this research are given a doctor's appointment for every 2 or 3 month. And so they end up checking their blood glucose once in 2 or 3 months respectively. Further, $\mathrm{HbA} 1 \mathrm{c}$ test, which is relatively expensive, is not requested or done as frequently as FBS. For these reasons, follow up appointments should be reduced to once in a month, at the most, rather than once in 2 or 3 months. On every of such visit, health care providers need to stress the benefits of regular SMBG, as well as adherence to other self-care practices. This is because increasing reach and frequency of appropriate message has an impact on behaviour change. ${ }^{29}$ In their study, Ayele and his colleagues $^{30}$ reported that patients who received information more frequently were more likely to adhere to self-care.

In line with the findings of Thungathurti and colleagues $^{31}$, majority of the patients in the present study knew the importance of having regular physical activity. However, they did not know that blood glucose should be measured before and after such activity, nor that it reduces the need for insulin or other diabetic drugs. Similar results have been reported by Adibe and associates. ${ }^{13,21}$ This is because very few of them actually exercise or carry out SMBG (as garnered by the researcher during interviews with respondents). Adherence with exercise has been shown to be very poor especially in developing countries. ${ }^{32}$ Moreover, these aspects of self-care (physical activity, SMBG) are not usually emphasised like areas such as diet and general medication-taking. Thus, the benefits of having regular physical activity, its effects on blood sugar (and hence on anti-diabetic drugs), and the need to carry out regular SMBG should constantly be stressed by health care providers.

For most patients, the doctor is at the centre of their care, and so whatever they say or do is assumed to be the best and irrevocable. This may explain why knowledge questions bordering on the opinion or decision of the doctor (only the doctors should make plans on how a person with diabetes can achieve his/her target goals; there should be mutual agreement between a person with diabetes and the doctor if he/she cannot change a particular lifestyle and afford his/her drugs; no person should check blood sugar and blood pressure of a diabetic patient except qualified medical doctor and other health personnel in the hospital) performed poorly. In an earlier study, eighty percent of diabetic patients clearly felt their general practitioner should solely be involved in their care and treatment. ${ }^{33}$ It would be enormously helpful to let patients know that their involvement is paramount for the successful care of diabetes. $^{34}$

\section{Factors affecting diabetes self-care knowledge}

The present study indicated that educational level of respondents was associated with knowledge. Previous studies have reported similar 
findings. ${ }^{21,28,35-37}$ A likely explanation is that those of a higher academic level (and hence of higher socioeconomic status) have a greater chance of obtaining knowledge from the mass media, books and the internet. In addition, they have fewer barriers in communicating with the health care team, and may have a good grasp of information. Expectedly, patients with no formal education were the least knowledgeable in this research.

Duration of diabetes was also found to be significantly associated with self-care knowledge. Similar findings have been recorded. ${ }^{13,21,27,38,39}$ With longer duration, there are more opportunities for exposure to information regarding diabetes. Repeated information or lessons about self-care in diabetes will eventually be grasped no matter how 'complex'. In addition, patients who have had the disease for some time might be experiencing some overt complications which will propel them to seek help, gaining more knowledge in the process. It is imperative therefore, to intensify trainings on selfmanagement for newly diagnosed patients and also for asymptomatic patients to prevent the development/reduce the progression of diabetes complications. However, education of diabetes and its care is a continuous process.

In contrast to the report of Adibe and colleagues ${ }^{21}$ which illustrated no relationship between monthly income and knowledge, the results of this study indicated a significant association of both variables. Patients with higher monthly income are likely to be able to afford their drugs and glucose meters, blood pressure monitoring machine, and other devices necessary for effective management of their disease. They are thus likely to know much about diabetes and attendant complications (such as hypoglycaemia, hyperglycaemia, and hypertension). Further, because majority of the respondents were graduates and civil servants (governmentemployed), they are likely to have been placed on an initial monthly income above NGN30,000, with annual increment (and occasional promotion) until retirement. From the age distribution, majority are still employed (only 4 retirees). The combined advantage of being highly educated, having relatively longer duration of diabetes post-diagnosis (because they would have discovered the disease earlier as they tend to go for regular medical checkups), access to self-care machines, and more opportunities for exposure to information, could explain the association of self-care knowledge and monthly income. Expectedly, the unsalaried were the least knowledgeable.

Negative attitude to disease condition was also shown to affect knowledge levels in this study. Similarly, low perceived seriousness of diabetes (negative attitude) has been identified as a factor affecting knowledge of self-care. ${ }^{21}$ Such attitude may be an unconscious rejection of a threat, or may reflect a lack of knowledge of the implications of diabetes for their health. ${ }^{33}$ Inappropriate health beliefs and attitudes concerning diabetes mellitus may make the patient uninterested in acquiring knowledge about the disease nor the associated self-care. A positive (favourable) attitude towards the disease, however, is likely to drive the patient to seek information concerning their disease. Diabetes educators/health-care providers have a vital role to play in the dissemination of knowledge of diabetes and its self-management and in so doing, change the inappropriate beliefs and attitude of patients with diabetes. Also, family, peer and support groups can influence patients' attitude and knowledge of selfmanagement.

\section{CONCLUSIONS}

Diabetes self-care knowledge was generally high among the sample studied, though some knowledge gaps were identified. To improve patients' knowledge of self-care and hence, active participation in their care, the reach and frequency of diabetes messages should be increased, and emphasis placed on the areas where knowledge was found to be inadequate. However, increase in such knowledge without accompanying improvement in adherence to self-care will be meaningless. To attain good health outcomes therefore, every available avenue to improve adherence should be exploited. Further, factors which predicted knowledge should be explored. With the above in place, patients are likely to have the best possible control of their blood glucose levels, with consequent reduction in the incidence and/or progression of diabetes-related complications, morbidity and mortality. These in turn will mean an improved quality of life and productivity, with decreased health care costs for the individual, their family, and the society at large.

\section{CONFLICT OF INTEREST}

None declared.

\section{CONOCIMIENTO SOBRE AUTO-CUIDADOS ENTRE LOS PACIENTES CON DIABETES TIPO 2 EN DOS ESTADOS DE NIGERIA}

\section{RESUMEN}

Objetivo: Evaluar el conocimiento de las prácticas de auto-cuidado, así como los factores responsables de ese conocimiento, entre pacientes con diabetes tipo 2 en dos estados de Nigeria.

Métodos: Se empleó un diseño de estudio descriptivo transversal. El estudio fue conducido en pacientes ambulatorios con diabetes tipo 2 que acudían a la consulta de endocrinología del Hospital de la Universidad de Uyo (UUTH) y al Hospital de la Universidad de Calabar (UCTH) entre junio-2012 y febero-2013. Para evaluar el conocimiento de las prácticas de auto-cuidado en la diabetes se utilizó el Diabetes Self-care Knowledge (DSCH-30). También se obtuvo la información sociodemográfica y la opinión de los respondentes sobre las posibles barreras al conocimiento sobre auto-cuidados. Se analizaron los datos usando Microsoft Excel y SPSS versión 14.0. La significación estadística para los análisis fue definida como valores de $\mathrm{p}$ menores de 0,05 .

Resultados: Se completaron y devolvieron un total de 303 de los 380 cuestionarios distribuidos (tasa de respuesta $=79,7 \%$ ). La mayoría de la muestra en estudio tuvo $70 \%$ o más de conocimiento global sobre autocuidados. El conocimiento sobre auto-cuidados estaba relacionado con el nivel de educación $(p<0,001)$, ingresos 
mensuales $(\mathrm{p}<0,001)$ y duración de la diabetes $(\mathrm{p}=0,008)$. La actitud negativa con la enfermedad fue el único factor asociado con el conocimiento (chi-cuadrado con un grado de libertad $=6,215 ; \mathrm{p}=0,013$ )

Conclusión: El conocimiento del auto-cuidado de la diabetes fue en general alto entre la población estudiada. El nivel educacional, los ingresos mensuales, la duración de la diabetes, y la actitud negativa ante la enfermedad predecían el nivel de conocimiento.

Palabras clave: Conocimientos, Actitudes y Práctica en Salud; Autocuidado; Diabetes Mellitus, Tipo 2; Nigeria

\section{References}

1. World Health Organization. Prevention of diabetes mellitus. Report of a WHO Study Group No. 844. Geneva: World Health Organization; 1994.

2. King H, Aubert RE, Herman WH. Global burden of Diabetes, 1995-2025. Diabetes Care. 1998;21(9):1414-1431.

3. International Diabetes Federation Diabetes Atlas (5th ed.) 2012 update. Available from: http://www.idf.org/sites/default/files/5E_IDFAtlasPoster_2012_EN.pdf (Accessed $5^{\text {th }}$ November, 2013).

4. Kasper L. Harrison's principle of internal medicine, 17th ed. Graw Hill Company: 2008.

5. Roglic G, Unwin N, Bennett PH, Mathers C, Tuomilehto J, Nag S, Connolly V, King H.The burden of mortality attributable to diabetes: Realistic estimates for the year 2000. Diabetes Care. 2005;28(9):2130-2135.

6. International Diabetes Federation 2011: Global burden of diabetes. IDF Diabetes Atlas, 5th edition. Available from: www.idf.org/diabetesatlas/se/the-global-burden (Accessed $26^{\text {th }}$ April, 2012).

7. Federal ministry of health in collaboration with WHO, EC, DFID. Standard treatment guidelines. Nigeria;2008:90-97.

8. Kaufman, A. Engaging Individuals with diabetes in self-care. MDPA Magazine 2012;20-21,29 Available from: www.dlife.com/mediakit/pdf/MDPA_article_pdf.pdf (Accessed18 ${ }^{\text {th }}$ May, 2012).

9. Ruchirawanitchathep W. [Diabetic patients' knowledge, attitude and self-care in Roumachi primary care unit]. Buddhachinaraj Medical Journal. 2008;25(1)60-66.

10. Inzucchi SE. Diabetic Facts and Guidelines.2011. Available from: www.endocrinology.yale.edu/patient (Accessed $26^{\text {th }}$ April, 2012).

11. Department of Health 2002. National service framework for diabetes: Standards. London: Department of Health; 2002.

12. American Diabetes Association. Clinical practice recommendations 2000. Diabetes Care. 2000;23(suppl 1):S1-116.

13. Adibe MO, Aguwa CN, Ukwe CV. The construct validity of an instrument for measuring type 2 diabetes elf-care knowledge in Nigeria. Trop J Pharm Res. 2011;10(5):619-629.

14. Ekpenyong CE, Akpan UP, Ibu JO, Nyebuk DE. Gender and age specific prevalence and associated risk factors of type 2 diabetes mellitus in Uyo metropolis, south eastern Nigeria. Diabetol Croat. 2012;41(1):17-28.

15. Adisa R, Fakeye TO, Fasanmade A. Medication adherence among ambulatory patients with type 2 diabetes in a tertiary healthcare setting in southwestern Nigeria. Pharm Pract (Granada). 2011;9(2):72-81.

16. Adebisi SA, Oghagbon K, Jimoh AK, Akande T, Olarinoye JK. Quality of diabetic care in a tertiary health care facility in Ilorin, Nigeria. Diabetol Croat. 2009;31:38-2.

17. Hassan AS, Al-Mousa ZA. Prevalence of obesity in patients attending diabetic care centres in Kuwait. Int Diabetes Digest. 1995;6:39-41.

18. Kamel NM, Badawy NA, El-Zeiny NA, Merdan IA. Sociodemographic determinants of management behaviour of diabetic patients Part II. Diabetics' knowledge of the disease and their management behaviour. East Mediterr Health J. 1999 Sep;5(5):974-983.

19. Burge S, White D, Bajorek E, Bazaldua O, Trevino J, Albright T, Wright F, Cigarroa L. Correlates of medication knowledge and adherence: Findings From the Residency Research Network of South Texas. Fam Med. 2005;37(10):712-718.

20. Marks J, Clark M. The hospital patient and his knowledge of the drugs he is taking. Int Nurs Rev. 1972;19(1):39-51.

21. Adibe MO, Aguwa CN, Ukwe CV, Okonta JM, Udeogaranya, Obinna P. Diabetes self-care knowledge among type 2 diabetic outpatients in south-eastern Nigeria. Int J Drug Dev Res. 2009;1(1):85-104.

22. Shah VN, Kamdar PK, Shah N. Assessing the knowledge, attitudes and practice of type 2 diabetes among patients of Saurashtra region, Gujarat. Int J Diabetes Dev Ctries. 2009;29(3):118-122. doi: 10.4103/0973-3930.54288

23. Nwankwo $\mathrm{CH}$, Nandy B, Nwankwo BO. Factors influencing diabetes management outcome among patients attending government health facilities in South East, Nigeria. Int J Trop Med. 2010;5(2):28-36.

24. Dunning T. Medication knowledge and self-management by people with type 2 diabetes. Aust J Adv Nurs. 2005;23(1):714.

25. Egede $L$, Xiaobou $Y$, Zheng D, Silverstein $M$. The prevalence and pattern of complementary and alternative medicine use in individuals with diabetes. Diabetes Care. 2002;25(2):324-329.

26. Dunning T. Complementary therapies and diabetes. Complement Ther Nurs Midwifery. 2003;9(2):74-80.

27. Moodley LM, Rambiritch V. An assessment of the level of knowledge about diabetes mellitus among diabetic patients in a primary healthcare setting. SA Fam Pract. 2007;49(10):16.

28. Abdo NM, Mohamed ME. Effectiveness Of Health Education Program For Type 2 Diabetes Mellitus Patients Attending Zagazig University Diabetes Clinic, Egypt. J Egypt Public Health Assoc. 2010;85(3-4):113-130.

29. Morowati A, Rouhani T. Diabetes self-care determinants model of diabetic patients referred to Yazd Diabetes Research Center. Daneshvar Medicine. 2009 Jun-Jul;16:81.

http://daneshvarmed.shahed.ac.ir/browse.php?a_id=57\&slc_lang=en\&sid=1\&ftxt=1 (Accessed $1^{\text {th }}$ Aug, 2014 
30. Ayele K, Tesfa B, Abebe L, Tilahun T, Girma E. Self Care Behavior among Patients with Diabetes in Harari, Eastern Ethiopia: The Health Belief Model Perspective. PLoS One. 2012;7(4):e35515. doi: 10.1371/journal.pone.0035515

31. Thungathurthi S, Thungathurthi S, Kumar VG. Self care knowledge on diabetes among diabetic patients in Warangal region. Int J Life Sci Pharma Res. 2012;2(2):16-21. Available from: http://ijlpr.com/admin/php/uploads/79_pdf.pdf (accessed 18th May, 2012).

32. Prabhu SS, Ramya N. Non-adherence to diabetic treatment and its effect on glycaemic control, study at a rural hospital of Tiruchirappalli, Tamilnadu, India. The Internet Journal of Health. 2011;2(2) Available from: http://ispub.com/lJH/13/1/8654 (Accessed 26th April, 2013).

33. Thompson AV, Neil HA, Thorogood M, Flower GH, Mann JI. Diabetes mellitus: attitudes, knowledge and glycaemic control in a cross-sectional population. J R Coll Gen Pract. 1988;38(315):450-452.

34. Walker R, Whittlesea C. (eds.). Clinical pharmacy and therapeutics (4th edition). London: Churchill Livingstone; 2002.

35. Abedini Z. Study of knowledge and practice of patient self directed care among diabetic patients. QOM University of Medical Science Journal. 2008;2(2). Available from: http://journal.muq.ac.ir/en/index.php/jmuqen/article/view/52 (accessed 17th May, 2012).

36. Kamel NM, Badawy YA, el Zeiny NA, Merdan IA. Sociodemographic determinants of management behaviour of diabetic patients. Part 1. Behaviour of patients in relation to management of their disease. East Mediterr Health $\mathrm{J}$. 1999;5(5):967-973.

37. Al-Qazaz HKh, Sulaiman SA, Hassali MA, Shafie AA, Sundram S, Al-Nuri R, Saleem F. Diabetes knowledge, medication adherence and glycemic control among patients with type 2 diabetes. Int J Clin Pharm. 2011;33(6):10281035. doi: 10.1007/s11096-011-9582-2

38. T'ang J, Chan C, Chan NF, Ng CB, Tse K, Lau L. A survey of elderly diabetic patients attending a community clinic in Hong Kong. Patient Educ Couns. 1999;36(3):259-270.

39. Priyanka Raj CK, Angadi MM. Hospital-based KAP study on diabetes in Bijapur, Karnataka. Indian Journal of Medical Specialities. 2010. Available from http://www.ijms.in/hospital-based-kap-study-on-diabetes-in-bijapur-karnataka.html (accessed 26th April, 2012).

40. Funnell MM, Haas LB. National standards for diabetes self-management education programs. Diabetes Care.1995;18(1):100-116. 